\title{
Save Vision Program: Federation of Islamic Medical Associations' Campaign Against Blindness
}

\author{
Aly A. Misha'l, MD, FACP \\ FIMA Executive Director \\ Senior Consultant in Endocrinology \\ Chief of Medical Staff \\ Islamic Hospital \\ Amman, Jordan
}

\begin{abstract}
The Federation of Islamic Medical Associations (FIMA) in 2005 initiated Save Vision, a special program to help the large number of blind people in the underprivileged countries of Africa and Asia. To date, thousands of eye surgeries, mostly for cataract removal, have been performed in several of these countries. In addition, training sessions for eye doctors, nurses, and paramedical personnel have been conducted, and eye hospitals and eye units in general hospitals have been established.
\end{abstract}

Key words: Blindness, developing countries, public health.

Introduction

$\Lambda$ ccording to World Health Organization (WHO) estimates, about 44 million people in the world are blind, with the largest concentration in the underprivileged countries of Africa and Asia. ${ }^{1}$ Blind people in these areas pose heavy social and financial burdens on their families, societies, and countries. Alleviation of this dilemma is instrumental in improving the quality of life and combating poverty for these individuals and the communities at large.

Visual impairment in the Eastern Mediterranean Region

World Health Organization statistics reveal widespread prevalence of vision problems in the eastern Mediterranean region. At least 6 million individuals are blind in this region, in addition to the 16-17 million people with various degrees of visual impair-

Correspondence should be directed to

info@islamic-hospital.org ment. 2

Seven countries in this region - Sudan, Somalia, Djibouti, Yemen, Palestine, Iraq, and Afghanistan are in dire need of combating visual impairment, according to WHO statistics. ${ }^{2}$

Cataracts are one of the major causes of visual impairment. ${ }^{2}$ However, a relatively simple surgery with implantation of a special lens results in an almost immediate cure. The backlog of cataract surgeries in Sudan alone is more than 500,000. Similar situations exist in other African and Asian countries.

In this paper, I will discuss the Save Vision program, initiated by the Federation of Islamic Medical Associations (FIMA) when its president led a FIMA medical group in a pilot project. Afterwards, in January 2005, FIMA launched Save Vision to combat blindness in some of these countries.

The FIMA Executive Committee adopted this initiative as an ongoing activity as long as the need exists. A steering committee was established and headquartered in Pakistan, where experienced and dedicated ophthalmologists have established a

JIMA: Volume 41, 2009 - Page 38 
record of excellence in combating visual impairment in South Asia.

Subcommittees of ophthalmologists were formed for various activities. A FIMA International Ophthalmic Committee was established, which included ophthalmologists who participated in FIMA Save Vision activities. In each country where the activity was conducted, a working group of FIMA eye doctors, local eye doctors, and official health authorities was formed.

In this major and continuous undertaking, FIMA collaborated with other organizations, especially the Arab Medical Union in Cairo, Egypt, the WHOEastern Mediterranean Region (WHO-EMRO), ministries of health, and local medical organizations in several African countries.

\section{Objectives of Save Vision Project}

Qualified volunteer eye doctors from several countries where FIMA has branches or collaborators have been deployed as groups to treat patients in eye camps. FIMA provided the special lenses, consumables, and surgical instruments. The main target is to alleviate cataracts. Decreasing the large backlog of cataract treatment requires years of diligent work.

Objectives of the Save Vision project include the following:

- Treatment of people suffering from visual impairment in needy, deprived communities, especially in remote areas.

- Training and capacity building of local eye doctors, nurses, and technicians

- Establishment of small, well-equipped eye hospitals or eye units in existing local hospitals to maintain the project.

\section{Treating Vision Problems in Target Countries}

The first objective of the Save Vision program is to treat vision problems in the target countries. Eye camps conducted in Africa involved Sudan, Somalia, Chad, Niger, Nigeria, Mali, and Senegal. At the time this article was written, more than 300,000 patients have been examined and more than 33,000 eye surgeries have been carried out with outstanding clinical outcomes. It has been extremely remarkable how successful the surgeries and how minimal the complications have been throughout the few years of this program.

In needy areas of Pakistan and Afghanistan, espe- cially among displaced people, Save Vision activities have been conducted with similar success. Plans are now underway to expand these programs in other African and Asian countries. More than 100 volunteer eye surgeons representing FIMA and collaborating organizations shouldered this activity.

\section{Training Providers to Save Vision}

The second dimension of the Save Vision initiative is training and capacity building of local eye doctors, nurses, and technicians so that they can intensify vision care activities throughout the year. Training took place during eye camps and was also conducted in Pakistani and Jordanian ophthalmology training centers for doctors and nurses from Sudan and Palestine. This crucial activity needs special care, attention, and promotion by all concerned parties.

\section{Building Treatment Centers}

The third focus of FIMA Save Vision is the establishment of eye hospitals or eye units in existing local hospitals in needy countries, especially in remote areas lacking medical services. The Federation was able to establish a well-equipped eye hospital in al-Genaina in West Darfur, an area that lacked an eye care facility. The building was provided by the Sudanese health authorities, and WHOEMRO extended a valuable helping hand by providing the salary of a Sudanese ophthalmologist appointed by Sudan's Ministry of Health and donating special lenses and consumables. Another FIMAsponsored eye hospital is being prepared, along the same lines, in the far eastern Sudanese province of al-Gadarif.

In the Palestinian West Bank and Gaza, classified by WHO as one of the most deficient countries in eye care, FIMA established and equipped one eye section in a local community hospital in the city of Toulkarm in the West Bank. Efforts are underway to do the same in other cities of the West Bank. The Federation of Islamic Medical Associations also provided the surgical instruments and other equipment for two eye hospitals in Gaza. The training of eye doctors and establishment of an ophthalmology eye training center are currently being planned.

\section{FIMA Save Vision: Lessons Learned}

The first attempts to establish eye hospitals in 
needy areas were helpful in improving plans to establish more eye hospitals and eye units in existing hospitals.

Local training and capacity building of eye doctors, nurses, and paramedics have proven to be crucial to medical and surgical work. Ultimately, it is the local qualified medical manpower that ensures the continuity and effectiveness of this initiative.

\section{Save Vision Activities}

Over the past 8 months, Save Vision completed the following projects, mostly focusing on cataracts:

- Dakar-Senegal, March 2008: Two Pakistani eye surgeons performed 350 eye surgeries.

- Butlam-Srilanka, April 2008: Four Pakistani eye surgeons performed 750 eye surgeries.

- Al-Genaina-Darfur-Sudan, May 10-20, 2008: Five eye surgeons from Pakistan, Jordan, and Sudan performed 500 surgeries.

- Al-Fashir-Darfur-Sudan, May 22 to June 3, 2008: Three surgeons from Pakistan and Jordan performed 350 surgeries.

- Aldaen-Sudan, June, 2008: Eye doctors from Pakistan, Jordan, and Sudan performed 540 surgeries.

- White Nile-Sudan, July 20-29, 2008: Five eye surgeons from Pakistan, Jordan, and Sudan performed 688 surgeries.

- Khartoum-Sudan, August 2008: Experienced senior ophthalmologists from Pakistan conducted oculoplastic surgery training for 52 Sudanese eye doctors and 30 paramedics.

- Katsina and Lagos-Nigeria, June 14-26, 2008: Eye surgeons from Pakistan and Jordan performed 750 eye surgeries. At the same time, 13 Nigerian eye doctors were trained in a workshop in Lagos and 12 were trained in a workshop in Katsina. Also, 35 paramedics were trained in a workshop in Lagos and 40 in a workshop in Katsina.

- Pakistan, January to August, 2008: Pakistani eye doctors performed 2000 eye surgeries, including 250 for inmates.

- Kasala-East Sudan, September 2008: Eye doctors from Pakistan, Jordan, and Sudan performed more than 500 eye surgeries.

- Malakal-South Sudan, October 2008: Five eye doctors from Pakistan, Jordan, and Sudan performed 140 eye surgeries. Adverse weather limited the number of procedures.

- Niger, November 2008: Three eye doctors from Pakistan performed 500 eye surgeries.

- Training workshop on corneal transplantation for 12 Sudani eye doctors, November 16-18, 2008, conducted by three senior ophthalmologists from Pakistan. Ten corneas were imported from Sri Lanka.

\section{References}

1. Magnitude and causes of visual impairment. Fact sheet $\mathrm{N}^{\circ}$ 282. World Health Organization. Available from http://www.who.int/mediacentre/factsheets/ fs282/en/. Updated 2004 Nov.

2. The work of WHO in the eastern Mediterranean region: annual report of the Regional Director. 1 January - 31 December 2003. World Health Organization: Regional Office for the Eastern Mediterranean. Available from http://www.emro.who.int/rd/AnnualReports/2003/. Updated 2004. 